\title{
Perturbing Inputs to Prevent Model Stealing
}

\author{
Justin Grana*† \\ Microsoft Corporation and Pardee RAND Graduate School \\ justin.grana@microsoft.com
}

May 13, 2020

\begin{abstract}
We show how perturbing inputs to machine learning services (ML-service) deployed in the cloud can protect against model stealing attacks. In our formulation, there is an ML-service that receives inputs from users and returns the output of the model. There is an attacker that is interested in learning the parameters of the ML-service. We use the linear and logistic regression models to illustrate how strategically adding noise to the inputs fundamentally alters the attacker's estimation problem. We show that even with infinite samples, the attacker would not be able to recover the true model parameters. We focus on characterizing the trade-off between the error in the attacker's estimate of the parameters with the error in the ML-service's output.
\end{abstract}

\section{Introduction}

In response to the advent of machine learning and artificial intelligence, several firms have developed cloud-based machine-learning as-a-service (ML-service) platforms [10. These platforms allow users to access a trained machine learning model by submitting queries and receiving output from the model. The main value of such services is that the end-users - individuals that are typically only interested in the model's output - do not need to preoccupy themselves with the model's details or the model training procedure.

As a simple example, an employee for a mortgage company might be interested in the probability that a potential customer defaults on their loan in the next year. The employee can upload characteristics of the customer such as age, educational attainment and outstanding debt to the ML-service. The pre-trained ML-service model will then output back to the employee the probability that the potential customer defaults on their mortgage. In this example, the employee receives the relevant information without having to understand the details of the underlying prediction model.

* (C)2020 IEEE. Personal use of this material is permitted. Permission from IEEE must be obtained for all other uses, in any current or future media, including reprinting/republishing this material for advertising or promotional purposes,creating new collective works, for resale or redistribution to servers or lists, or reuse of any copyrighted component of this work in other works.

${ }^{\dagger}$ I would like to thank Gavin Hartnett, Andrew Lohn, Adrienne Propp, A. Christian Johnson, Brian Vegetabile and other participants at the AI study circle for their feedback and comments. The paper greatly improved because of them. 
Unfortunately, these models are subject to several types of attacks [12, 16, 9]. One particularly prevalent type of attack is one in which an attacker conducts a model-stealing attack [13, 14, 5, 8, 4, 17. In such an attack, a malicious actor attempts to learn the underlying model of the MLservice. In this paper, we present a method for protecting against model stealing attacks where the attacker is directly interested in learning the parameters of the model. Our method relies on strategically adding noise to the inputs of the machine learning model which introduces endogeneity into the attacker's estimation problem, and thus an attacker's estimate of the model's parameters are biased and inconsistent [1, 15, 2. Endogeneity arises in a regression model when the error term is correlated with one or more of the regressors. In models with endogeneity, even with infinite samples the attacker cannot learn the true parameters of the trained model. The main trade-off our method considers is how much noise in the output of the ML-service is needed to induce a bias of a certain size into the parameter estimates. This trade-off is similar to other methods aimed at preventing model stealing [6], however ours is the first to consider the noise in the output as a function of noise in the input.

There are several reasons why an attacker would have an incentive to steal the ML-service model parameters. First, ML-services usually charge per query. Therefore, if a user can learn the parameters of an ML-service model, they can build their own prediction API and no longer have to pay for queries. Alternatively, if the data a user submits to the platform is sensitive, then the user might wish to build its own model to bypass any security concerns associated with sending queries over a network.

Yet another reason ML-service parameters are subject to stealing is because attackers have an inherent value in knowing the parameters. For example, consider an ML-service platform that reports the probability that an individual purchases a product conditional on whether the individual saw a targeted internet advertisement. A firm that is considering placing internet advertisements might be interested in stealing the model to learn the parameters that determine the average treatment effect (ATE) of the advertisement. Learning this parameter would inform the firm how much they would be willing to pay for targeted advertising.

Our method protects against both an attacker that steals the ML-service's parameters with the intention of replicating the ML-service as well as an attacker that has an inherent value in learning the parameters. As a point of clarification, our method does not directly address the case in which an attacker wants to steal the ML-service to replicate its prediction power but does so by other means than parameter stealing. For example, we don't explicitly address how our method protects against an attacker that builds a neural network to replicate the ML-service model to leverage its predictive power only with no concern for the actual ML-service parameters. Nevertheless, our method still protects against a broad class of attackers.

It is also crucial to emphasize that this initial proof of concept is simplified in two main ways. First, we only consider linear and logistic regression ML-services. The properties of endogeneity do indeed extend to more sophisticated models, including neural networks $\underline{3}$, but using such techniques would unnecessarily complicate the analysis and detract from the key implications of adding noise to ML-service inputs. Secondly, we only consider relatively simple attacker models. A natural extension would be to include a full game-theoretic formulation complete with attacker and defender utility functions. While that is a valuable extension, this work is meant to illustrate the efficacy of a defender whose strategy set contains options on how to add noise to ML-service inputs. 


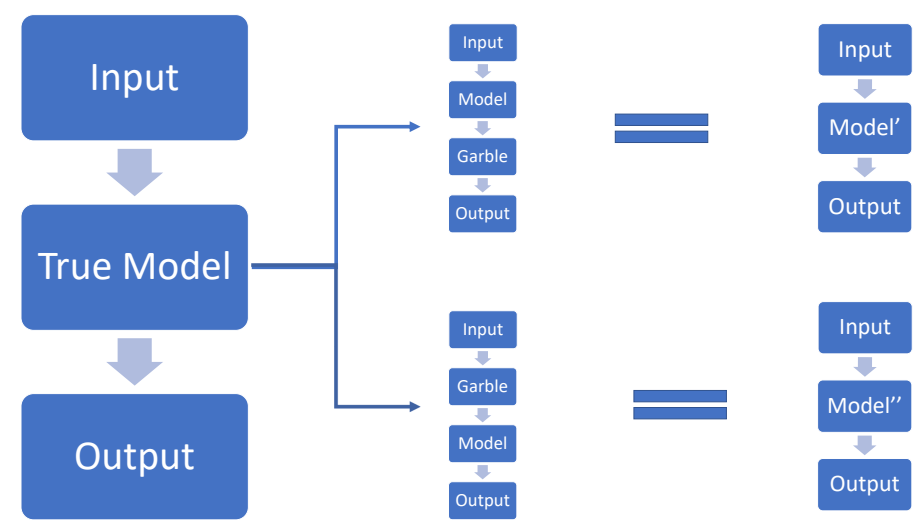

Figure 1: Two approaches to perturbing models

\section{Related Work}

The literature on model stealing is relatively young. Initial research showed that if an ML-service is a regression model and the attacker knew the functional form of the model, it would be able to recover the model parameters if it submitted one query per model parameter [13. Other work included methods for stealing neural networks and decision tree models from ML-services [13, 11] as well as model hyper parameters [14. Methods to protect against model stealing are also a topic of interest, where most focus on selectively perturbing the output of the ML-service [6]. Output perturbations include rounding the output or truncating the output to obfuscate the ML-service's true output. 1

Figure 1 contrasts our approach with the literature. In the figure, the leftmost vertical sequence represents an ML-service without any protection against model stealing. In other words, inputs are submitted to the service and the service returns an output. The top path is the traditional method of preventing model stealing by perturbing the output. This is the case when random noise is added to the output [6], or instead of returning class probabilities, the ML-service only returns a label 77. What such a transformation effectively does is transforms the ML-service's "True Model" $(M)$ to another model, Model' $\left(M^{\prime}\right)$. However, perturbations to the outputs do not guarantee that $M$ 's parameters cannot be known simple by sampling $M^{\prime}$. For example, if the only difference between $M$ and $M^{\prime}$ was that $M^{\prime}$ adds a mean zero normal random variable to the output of $M$, an attacker would be able to learn the parameters of $M$, just by querying $M^{\prime}$ sufficiently many times.

Our approach is similar to the literature in that by perturbing the inputs we are changing the ML-service's model from $M$ to Model" $\left(M^{\prime \prime}\right)$, as shown in the bottom path of figure 1. However, our approach illuminates several novelties that were obscured in the case of perturbed outputs. First, we introduce a simple garbling function that makes it impossible for the attacker to learn the parameters of $M$, even with infinite samples of $M^{\prime \prime}$. This is an improvement over the case when mean-zero noise is added to the ML-services output. Secondly, we show that in non-linear models, it is possible to perturb the inputs to obscure the parameters from the attacker while maintaining a negligible difference in the output $M$ and $M^{\prime \prime}$. Finally, we show how to leverage the correlation

\footnotetext{
${ }^{1} \mathrm{~A}$ small body of research focuses on detecting adversarial patterns in a user's sequence of queries [5]
} 
structure of the inputs to further reduce the difference between the outputs of $M$ and $M^{\prime \prime}$ while still obscuring the true parameters from the attacker. Since previous models focus only on perturbing outputs, this notion of leveraging the structure of inputs to prevent model stealing has yet to be explored. Of course, with infinite samples the attacker would be able to approximate $M^{\prime \prime}$. However, we are interested in the case where the defender wants to obscure the parameters of $M$, not $M^{\prime \prime}$ and we examine how much noise the defender must tolerate in order to obscure the parameters of $M$ by a given amount.

\section{General Framework}

A machine learning model is a function $f_{\theta}: \mathcal{X} \rightarrow \mathcal{Y}$. The function $f_{\theta}$ is from $\mathbb{R}^{K} \rightarrow R^{M}$, and is governed by parameters $\theta \in \Theta \in \mathbf{R}^{P}$. If the ML-service did not need to be protected from model stealing, a user would submit a vector $\mathbf{x}$ and the ML-service would return the value $f_{\theta}(\mathbf{x})$. Previous work on preventing model stealing focuses on changing the function $f_{\theta}$ so that when an attacker submits a vector $\mathbf{x}$, the ML-service returns $\tilde{f}_{\tilde{\theta}}(\mathbf{x})$. Our approach is different. Instead of changing $f$ directly, we change $\mathbf{x}$ before it goes into the machine learning service. Specifically, there is a garbling function $g: \mathcal{X} \rightarrow \mathcal{X}$ such that when a user submits an input $\mathbf{x}$, the ML-service returns $f_{\theta}(g(\mathbf{x}))$.

An attacker submits a dataset $\mathbf{X}=\left\{\mathbf{x}_{1}, \mathbf{x}_{2} \cdots \mathbf{x}_{N}\right\} \in \mathcal{X}^{N}$ to the machine learning model and receives in return the predictions $\hat{\mathbf{Y}}=\left\{f\left(g\left(\mathbf{x}_{1}\right)\right), f\left(g\left(\mathbf{x}_{2}\right)\right), \cdots f\left(g\left(\mathbf{x}_{N}\right)\right)\right\} \in \mathcal{Y}^{N}$. Although not necessary, we constrain $g$ such that for some dataset $\mathbf{X}$, the expected deviation between $\mathbf{x}$ and $g(\mathbf{x})$ is 0 . Specifically

$$
\frac{1}{|\mathbf{X}|} \sum_{\mathbf{x} \in \mathbf{X}} \mathbf{E}_{g}[\mathbf{x}-g(\mathbf{x})]=0
$$

Again, this is not a necessary constraint but we only include it to show how our method is valid under mean-zero expected error, which might be a desirable property. Of course, loosening this constraint does not degrade the performance of our method. In addition, our method does not rely on the attacker submitting an entire dataset at once. Specifically, our method is also valid when an attacker submits sequential queries. However, in that case the average error between the true inputs and the garbled inputs would not, in general, be 0. Again, this does not degrade the performance of our method.

For all $N$, an attacker has an estimating function $h: \mathcal{X}^{N} \times \mathcal{Y}^{N} \rightarrow \Theta$ that maps the attacker's inputs and outputs to its estimate of the parameters, $\hat{\theta}$. Common estimating functions are the least squares or maximum likelihood estimates. To evaluate the effectiveness of our method we consider two quantities:

1. The difference between the attacker's estimate of the parameters and the true parameters with infinite samples, which is given by

$$
\mathcal{D}=\operatorname{plim} h(\mathbf{X}, \hat{\mathbf{Y}})-\theta
$$

We call this the attacker's "estimation error".

2. The expected squared difference between the ML-service's output without perturbing the input and the ML-service's output with the perturbed output, which is given by

$$
\sigma^{2}=\mathbf{E}\left[\left(f_{\theta}(\mathbf{x})-f_{\theta}(g(\mathbf{x}))\right)^{2}\right]
$$


We call this the ML-service's "prediction error." However, it is not the difference between what the ML-service predicts and some true value but instead is the difference between what the ML-service predicts when it garbles the inputs and what the ML-service would predict if it didn't garble to inputs.

The equation for $\mathcal{D}$ is the difference between the true parameters and the asymptotic limit of the attacker's estimate. The second term is the mean squared error between the ML-services output and what the ML-service would output if it wasn't concerned with protecting against model stealing. Broadly speaking, the goal is to make $\mathcal{D}$ as high as possible while minimizing $\sigma^{2}$. The intuition is that $\mathcal{D}$ is the error in the attacker's estimate of the true parameters, which is what is used to prevent model stealing. However, the ML-service still needs to be useful to normal users so it can't distort the output so much that the ML-service becomes useless. The ML-service is most useful when $\sigma^{2}$ is low.

One subtlety is that if the attacker knew the value of $\mathcal{D}$, it would be able to adjust its estimates to correct for the error. Therefore, higher values of $\mathcal{D}$ would not necessarily prevent model stealing. In a full game theoretic formulation, the defender would likely randomize over values of $\mathcal{D}$ so that the attacker wouldn't be able to know exactly how to correct for the bias in its estimates. Since in this proof of concept, we do not consider the full game theoretic effects, we motivate high values of $\mathcal{D}$ through its deterrent effect. Specifically, if the attacker knew that the defender could obfuscate the parameters with a high degree of error, then a high value of $\mathcal{D}$ would disincentivize the attacker from stealing the model. In other words, the attacker might not know exactly the value of $\mathcal{D}$ but knows that the defender could obfuscate the estimates up to a certain amount and thus, the attacker would not be willing to steal the model because it knows that it's final estimates might be prohibitively inaccurate.

\section{Results}

In this section we present a mix of analytical and numerical results. We begin with the simplest case of a one covariate linear regression model. The linearity of the model permits an analytical analysis. We then extend the model to the case of logistic regression and examine our method's performance We conclude with an analysis of correlated regressors.

\subsection{Simple Linear Regression}

In the simplest case, consider the one variable linear machine learning model $f_{\theta}=\alpha+\beta x$ where $\theta=\{\alpha, \beta\}$. Given a dataset $\mathbf{X}$ of queries, to induce endogeneity we define the garbling function of a specific input $x$ as

$$
g(x)=x+\gamma\left(\mu\left(x, \lambda^{2}\right)\right)
$$

where $\gamma$ and $\lambda$ are constants and $\mu(a, b)$ is a normal random variable with mean $a$ and variance $b$. Given the dataset $\mathbf{X}, \hat{\mathbf{Y}}$, the attacker's estimation function, $h$, is the ordinary least squares solution. That is, the function $h$ for the attacker is "choose the parameters $\hat{\alpha}$ and $\hat{\beta}$ that minimizes $\sum_{i=1}^{N}\left(\hat{y}_{i}-\left(\hat{\alpha}+\hat{\beta} x_{i}\right)\right)^{2}$, where $\hat{y}$ is the value returned from the ML-service. Since $\beta$ gives the marginal impact of a change in $x$, we focus the rest of the analysis on the slope parameters and ignore the intercept. 
Before deriving the terms for $\mathcal{D}$ and $\sigma$, it is important to emphasize that this "simple" formulation is not as restrictive as it seems. First, if instead of using least squares, the attacker used a maximum likelihood estimation procedure, the results would not change. Secondly, the restriction of only one regressor is also not as restrictive as it seems. For example, if there were multiple uncorrelated regressors and only one of the regressors was perturbed, the results would not change. Therefore, this simple case actually covers multiple linear regression when one regressor is garbled. Later, we consider the case of multiple perturbations of correlated regressors.

We now derive the relationship between $\mathcal{D}$ and $\sigma^{2}$ in the linear model.

Proposition 1 (Large Sample Results). The relationship between the error in the attacker's estimate and the root mean square error is given by:

1. $\mathcal{D}=\operatorname{plim}(\hat{\beta})-\beta=\beta \gamma$

2. $\sigma^{2}=\left[(\beta \gamma)^{2}\left(\operatorname{Var}(x)+\lambda^{2}\right)\right]$

Proof. First we prove 1. Under the garbling function $g$, the data generating process for the MLservice is

$$
\begin{aligned}
\hat{y} & =\alpha+\beta(x+\gamma(\mu(x, \lambda))) \\
& =\alpha+\beta(x+\gamma(x+\lambda \epsilon)) \\
& =\alpha+(1+\gamma) \beta x+\beta \lambda \gamma \epsilon \\
& =\alpha+\tilde{\beta} x+\eta
\end{aligned}
$$

where $\tilde{\beta}=(1+\gamma) \beta, \epsilon$ is a standard normal random variable and $\eta$ is a composite mean-zero noise term. This shows that the data generating process for the ML-service is a simple linear model in $x$. Therefore, when the attacker estimates $\hat{\beta}$ from the data it estimates a linear regression model with slope parameter equal to $\tilde{\beta}$. Because OLS estimates converge to the true parameters of the data generating process, the attacker's estimate converges to $\tilde{\beta}$ and thus $\tilde{\beta}-\beta=\beta \gamma$.

To prove part 2 of proposition 1 , note that

$$
\begin{aligned}
\sigma^{2} & =\mathbf{E}\left[\left(f_{\theta}(\mathbf{x})-f_{\theta}(g(\mathbf{x}))\right)^{2}\right]=\mathbf{E}\left[(y-\hat{y})^{2}\right] \\
& =\mathbf{E}\left[(\alpha+\beta x-(\alpha+\beta(1+\gamma) x+\beta \gamma \lambda \epsilon))^{2}\right] \\
& =\mathbf{E}\left[\left((\beta \gamma)^{2} x^{2}+2 \beta^{2} \gamma^{2} \lambda x \mu+(\gamma \beta \lambda \epsilon)^{2}\right)\right] \\
& =\left[(\beta \gamma)^{2} \mathbf{E}\left[x^{2}\right]+2 \beta^{2} \gamma^{2} \lambda \mathbf{E}[x \mu]+(\gamma \beta \lambda)^{2} \mathbf{E}\left[\epsilon^{2}\right]\right] \\
& =\left[(\beta \gamma)^{2}\left(\operatorname{Var}(x)+\lambda^{2}\right)\right]
\end{aligned}
$$

where the last line follows because $x$ and $\epsilon$ are independent and $\epsilon$ is a standard normal variable.

Proposition 1 gives the precise trade-off between the attacker's error in the parameter estimates and the noise in the output required to induce the estimates. The proposition reveals several high level features. First, the error in the attacker's estimate is linear in $\beta$ and $\gamma$. That means the attacker's relative error is linear in $\gamma$ only. Second, the prediction error depends on the variance of $x$. This is an artifact of having systematic errors. Specifically, larger absolute values of $x$ lead 
to higher errors because larger values of $x$ are perturbed more. Although the linear relationship between the prediction error and the variance of $x$ is a product of the functional form of the garbling function, the main insight is that the prediction error will depend on the distribution of $x$ because endogeneity requires systematic errors.

Finally, the proposition shows that the prediction error is increasing in the free parameter, $\lambda$. Since the goal is to minimize $\sigma^{2}$, an obvious choice is to set $\lambda$ to 0 . However, there are several exogenous reasons why the defender would not do this. First, $\lambda$ enters the equation for the attacker's estimation error in finite samples as given in the following proposition:

Proposition 2 (Small Sample Results). Suppose the attacker submits inputs of size $n$ and estimates $\beta$ using least squares or maximum likelihood. Then:

$$
\mathbf{E}\left[(\beta-\hat{\beta})^{2}\right]=(\beta \gamma)^{2}+(\beta \gamma \lambda)^{2} \mathbf{E}\left[\left(\sum_{i=1}^{n} x_{i}^{2}\right)^{-1}\right]
$$

Proof. The true data generating process with garbling is given by:

$$
\hat{y}=\alpha+(1+\gamma) \beta x+\beta \lambda \gamma \mu
$$

which is a standard linear regression model whose finite $n$ distribution is given in [1] chapter 4

Consequently, if the defender is interested in not just the infinite sample limit but also the smallsample parameter estimates, changing $\lambda$ gives the defender another lever to obfuscate the model. Secondly, the defender might also be interested in protecting the ML-service from being replicated by an attacker (i.e. protecting an attacker from stealing $M^{\prime \prime}$ ). If $\lambda=0$, then the ML-service is deterministic and it is relatively simple for an attacker to replicate the ML-service. Therefore, setting $\lambda>0$ protects against stealing $M^{\prime \prime}$. Finally, from a strategic perspective, a sophisticated attacker would form beliefs about the garbling function and the associated parameters $(\gamma$ and $\lambda)$ in order to best reconstruct an estimate for $\beta$. If the defender always sets $\lambda=0$, then the attacker only needs to reason about $\gamma$ to make inference about the value of $\beta$, which reduces the dimensionality of the attacker's decision problem.

Finally, if the attacker knew the nonzero value of $\lambda$, it would be able to recover $\beta$, as given by the following proposition.

Proposition 3. Suppose the attacker knows the value of $\lambda$ and the attacker estimates the regression coefficients $\hat{\beta}$ and $\hat{\alpha}$ and the model variance $\hat{\Sigma}^{2}=\sum_{i=1}^{N}\left(\hat{y}-\left(\hat{\alpha}+\hat{\beta} x_{i}\right)\right)^{2}$ using least squares or maximum likelihood. Then in the infinite limit, the attacker can identify $\beta$ as $\hat{\beta}-\frac{\hat{\Sigma}}{\lambda}$.

Proof. As stated in proposition 1, $\hat{\beta}$ converges to $(1+\gamma) \beta$. Since the attacker is estimating a linear model, the attackers estimate of the error $\hat{\Sigma}^{2}$ converges to the error in the true model which is given by $(\beta \gamma \lambda)^{2}$ in equation 5 . This gives the following two equations where the unknowns are $\beta$ and $\gamma$ :

$$
\begin{aligned}
& \hat{\Sigma}^{2}=(\beta \gamma \lambda)^{2} \\
& \hat{\beta}=\beta(1+\gamma)
\end{aligned}
$$

Solving for $\beta$ yields

$$
\beta=\hat{\beta}-\frac{\hat{\Sigma}}{\lambda}
$$

and thus $\beta$ is identified. 
Proposition 3 illustrates how for our method to be effective, a sophisticated attacker cannot know $\lambda$. If it did, it would be able to recover the true value of $\beta$. Assuming $\lambda>0$, this means in a full game theoretic treatment the defender would likely randomize the values of $\gamma$ and $\lambda$ so that the attacker couldn't recover $\beta$ from the data. ${ }^{2}$

\subsection{Logistic Regression}

Injecting endogeneity is not unique to the linear model and also applies to nonlinear models. The logistic regression model is a special type of regression model that takes inputs and outputs $\hat{y} \in[0,1]$ and is a common tool to predict the input's "class." For example, logistic regression can be used to model the probability of recidivism, the probability an individual files bankruptcy, the probability a computer network is under attack or in the multinomial case, the probability that an image contains each of the digits 0-9.

Under the same garbling function, $g$, as before, the logistic ML-service model with perturbed inputs (assuming the 0 mean for $x$ ) is given by:

$$
\begin{aligned}
\hat{y} & =\frac{e^{\alpha+\beta g(x)}}{1+e^{\alpha+\beta g(x)}} \\
& =\frac{e^{\alpha+\beta(x+\gamma(x+\lambda \mu))}}{1+e^{\alpha+\beta(x+\gamma(x+\lambda \mu))}}
\end{aligned}
$$

Since the binomial logistic regression model can be transformed into a linear model that predicts the "log odds", the analysis is the same as in section 4.1. However, when translating the log-odds back to probabilities, the results are slightly different as given in figure 2 The figure shows that it is possible to obscure the true value of the parameters with a minimal effect on prediction error. This is due to the systematic perturbation of the inputs. Specifically, the perturbation of the inputs are most extreme for inputs that are high in absolute value and mild for inputs low in absolute value. Crucially, those larger perturbations occur along a relatively flat portion of the logistic curve. Therefore, the marginal effect of perturbing the inputs at high absolute value inputs is minimal. Of course, the prediction error in the log-odds is still quadratic in $\gamma$, as proposition 1 established and figure 2 shows, but after transforming the log odds back to the probabilities of interest, the prediction error is negligible.

Undoubtedly, this result is due to the functional form of $g$. For example, if $x$ values along the steepest part of the logistic curve were perturbed the most, then different results would emerge. While determining what the optimal functional form of $g$ should be is indeed an important and broad question, these results show that in nonlinear models, certain choices of $g$ can allow the defender to induce error in the parameter estimates with minimal impact on the prediction error. Specifically, perturbing inputs where the derivative is close to zero provides minimal impact on the prediction error.

\subsection{Multiple Perturbations}

Finally, we analyze the linear model with multiple regressors where each regressor is garbled. In this exposition, we consider the bivariate case, though the results are easily generalized to $K$ regressors.

\footnotetext{
${ }^{2}$ Another possibility would be to also add a mean-zero noise term to the output. So for example, suppose the defender garbles with $g$ but also adds $\mu\left(0, \lambda_{2}^{2}\right)$ to the output. Then the equations for $\hat{\Sigma}$ and $\hat{\beta}$ would contain the variables $\beta, \gamma, \lambda$ and $\lambda_{2}$. Therefore, as long as the attacker doesn't know any 2 of the variables, it would not be able to recover $\beta$.
} 


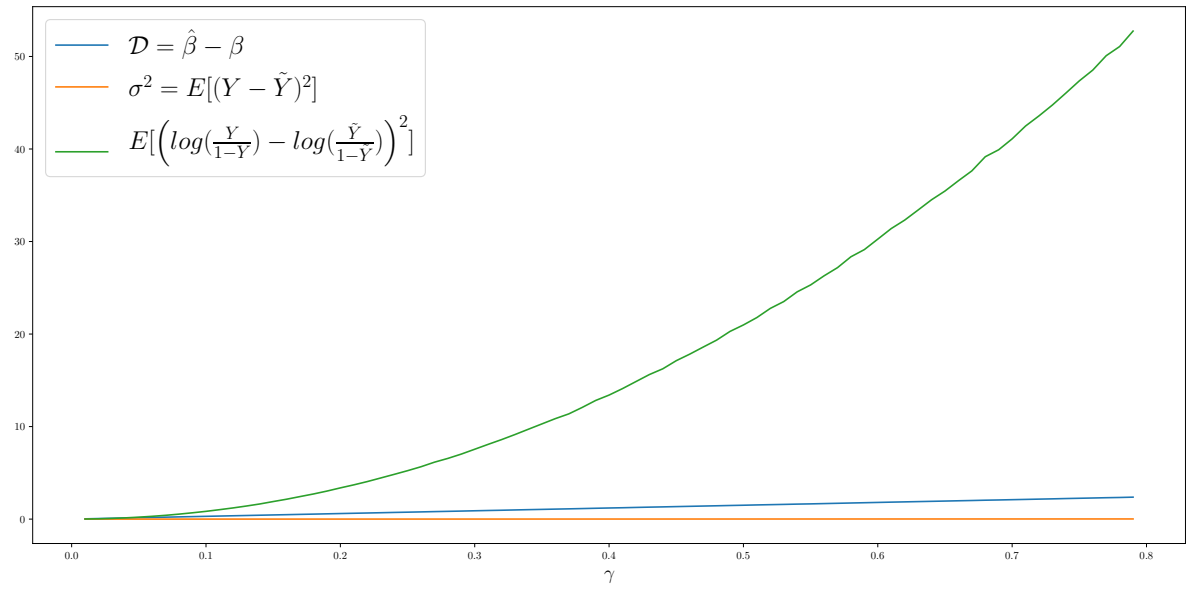

Figure 2: The figure shows that while the prediction error for the $\log$ odds is exponential in $\gamma$, the prediction error in the actual predicted probabilities is minimal. This is due to the functional specification in the systematic errors in $X$. For this plot, $\alpha=2, \beta=3, N=100000, \lambda=1, \operatorname{Var}(x)=$ 8.3

Specifically we consider the ML-service where $\hat{y}=f_{\theta}=\alpha+\beta_{1} x_{1}+\beta_{2} x_{2}$. We now also perturb each $x_{i}$ independently so that

$$
g\left(x_{i}\right)=x_{i}+\gamma_{i}\left(\mu_{i}\left(x_{i}, \lambda\right)\right)
$$

where we assume the mean of $x_{i}$ is 0 . We could also of course let $\lambda$ depend on $i$ but for ease of notation, we assume $\lambda$ is the same for each regressor. Like in the previous section, we assume the attacker estimates the parameters using either least squares of maximum likelihood. We now establish the multivariate version of proposition 1 .

Proposition 4. The relationship between the error in the attacker's estimate and the root mean square error is given by:

1. $\operatorname{plim}\left(\hat{\beta}_{i}\right)-\beta_{i}=\beta_{i} \gamma_{i}$

2. $\sigma^{2}=\left[\left(\beta_{1} \gamma_{1}\right)^{2} \operatorname{Var}\left(x_{1}\right)+\left(\beta_{2} \gamma_{2}\right)^{2} \operatorname{Var}\left(x_{2}\right)\right.$

$+2 \beta_{1} \beta_{2} \gamma_{1} \gamma_{2} \operatorname{COV}\left(x_{1}, x_{2}\right)+$

$\left.\left(\lambda\left(\gamma_{1} \beta_{1}+\gamma_{2} \beta_{2}\right)\right)^{2}\right]$

where $\operatorname{COV}(x, y)$ is the covariance between random variables $x$ and $y$.

Proof. As in the proof for proposition 1, it is straightforward to show that the ML-service's regression model with the perturbed inputs is given by

$$
\hat{y}=\alpha+\left(1+\gamma_{1}\right) \beta_{1} x_{1}+\left(1+\gamma_{2}\right) \beta_{2} x_{2}
$$




$$
+\lambda\left(\beta_{1} \gamma_{1}+\beta_{2} \gamma_{2}\right) \epsilon
$$

where $\epsilon$ is a standard normal random variable. By the same argument as in proposition 1, the attacker estimates of $\beta_{i}$ are multiplied by a factor of $\left(1+\gamma_{i}\right)$. Proving 2 can be derived as follows:

$$
\begin{aligned}
& \sigma^{2}= \mathbf{E}\left[\left(f_{\theta}(\mathbf{x})-f_{\theta}(g(\mathbf{x}))\right)^{2}\right]=\mathbf{E}\left[(y-\hat{y})^{2}\right] \\
&= \mathbf{E}\left[\left(\alpha+\beta_{1} x_{1}+\beta_{2} x_{2}-\right.\right. \\
&\left(\alpha+\beta_{1}\left(1+\gamma_{1}\right) x_{1}+\beta_{2}\left(1+\gamma_{2}\right) x_{2}+\right. \\
&\left.\left.\left.\lambda\left(\beta_{1} \gamma_{1}+\beta_{2} \gamma_{2}\right) \epsilon\right)\right)^{2}\right] \\
&=\mathbf{E}\left[\left(\left(\beta_{1} \gamma_{1}\right)^{2} x_{1}^{2}+\left(\beta_{2} \gamma_{2}\right)^{2} x_{2}^{2}+2 \beta_{1} \beta_{2} \gamma_{1} \gamma_{2} x_{1} x_{2}+\right.\right. \\
&\left.\left.\left(\lambda\left(\beta_{1} \gamma_{1}+\beta_{2} \gamma_{2}\right) \epsilon\right)^{2}\right)\right] \\
&=\left[\left(\beta_{1} \gamma_{1}\right)^{2} \mathbf{E}\left[x_{1}^{2}\right]+\left(\beta_{2} \gamma_{2}\right)^{2} \mathbf{E}\left[x_{2}^{2}\right]+\right. \\
& 2 \beta_{1} \beta_{2} \gamma_{1} \gamma_{2} \mathbf{E}\left[x_{1} x_{2}\right]+ \\
&\left.\left(\lambda\left(\beta_{1} \gamma_{1}+\beta_{2} \gamma_{2}\right)\right)^{2} \mathbf{E}\left[\epsilon^{2}\right]\right] \\
&=\left[\begin{array}{l}
\left(\beta_{1} \gamma_{1}\right)^{2} \operatorname{Var}\left(x_{1}\right)+\left(\beta_{2} \gamma_{2}\right)^{2} \operatorname{Var}\left(x_{2}\right)+ \\
2 \beta_{1} \beta_{2} \gamma_{1} \gamma_{2} \operatorname{COV}\left(x_{1}, x_{2}\right)+ \\
\left.\left(\lambda\left(\beta_{1} \gamma_{1}+\beta_{2} \gamma_{2}\right)\right)^{2}\right]
\end{array}\right.
\end{aligned}
$$

where the last line follows because $x_{i}$ and $\epsilon$ are independent and $\epsilon$ is a standard normal variable 3

The key difference between the simple univariate regression model and the multiple regression case is that the ML-services prediction error is influenced by the covariance of the regressors. This leads to several insights. First, if the covariance is 0 and the regressors are uncorrelated, then the prediction error is the sum of the prediction errors when only one of the regressors is perturbed. More importantly, for a non-zero covariance, the prediction error can be less than the sum of the prediction error when only one variable is perturbed.

To understand how the covariance of $x_{1}$ and $x_{2}$ impacts the prediction error consider the following example. Suppose that $\beta_{1}, \beta_{2}, \gamma_{1}$ and $\gamma_{2}$ are positive and $x_{1}$ and $x_{2}$ are negatively correlated so that the covariance is less than 0 . Also, recall how the garbling function works. In general, the perturbations are such that, on average, positive values of $x_{1}$ and $x_{2}$ are increased and negative values are decreased. Furthermore, due to the negative covariance, when $x_{1}$ is relatively high (and

\footnotetext{
${ }^{3}$ It is straightforward to show that for the $K$ variate case, $\sigma^{2}=\Gamma^{T} \Sigma \Gamma+\lambda^{2}\|\Gamma\|_{2}^{2}$ where $\Gamma$ is a $K \times 1$ vector where the $i$ 'th element is $\beta_{i} \gamma_{i}$ and $\Sigma$ is the covariance matrix of $X$.
} 
positive), $x_{2}$ is relatively low (negative). So, when a high value of $x_{1}$ is perturbed, it is, on average, increased. Since, by assumption, $\beta_{1}$ is positive, the estimated output of $y$ would be above the predicted value when there is no perturbation. However, at the same time $x_{2}$ is perturbed and, on average, decreased. Since by assumption, $\beta_{2}>0$, the estimated output of $y$ would be below the predicted value when there is no perturbation. Or in other words, the perturbation in $x_{2}$ cancels out some of the error due to the perturbation in $x_{1}$.

The impact of the covariance is not limited to the case of negative covariances. Since $\gamma_{1}$ and $\gamma_{2}$ are free parameters, a defender that sets $\gamma_{1}$ and $\gamma_{2}$ to ensure a certain relative prediction error can choose the sign of $\gamma_{1}$ and $\gamma_{2}$ so that the relative error in the parameter estimate is unchanged but $\gamma_{1} \gamma_{2} \beta_{1} \beta_{2} \operatorname{COV}\left(x_{1}, x_{2}\right)<0$. This ensures that the defender leverages the reduction in prediction error due to correlated inputs.

\section{Discussion and Future Work}

We used regression models to show that it is possible to strategically inject error into an ML-service's inputs to guard against an attacker learning the model parameters. Importantly, our scenario is different from other scenarios in the literature as in our case, we assume the attacker is interested in recovering the ML-service's parameters and not only replicating the output of the ML-service.

For the univariate linear regression model, we introduced a simple garbling function that transformed the inputs before being sent to the ML-service. We illustrated the trade-off between the attacker's error in the estimation of the model's parameters and the error in the machine learning model's output against what its output would be if it didn't perturb the inputs. Under our garbling function, the (squared) prediction error scales quadratically in the error of the estimate.

We showed that our results extend to the case of logistic regression. However, while the scaling of the prediction and estimate error are the same when considering the log-odds ratios, the relationship does not hold when considering the error in the outputted probability. Specifically, since our garbling function injects most of the noise in the tails of the logistic curve, the garbling has little effect on the prediction error of the ML-service. While this is a simple example and will likely not replicate for all types of ML-services, it shows that carefully chosen garbling functions in non-linear models may perform better than the same garbling function in a linear model. Finally, we showed how a defender can leverage correlation among the inputs to reduce the ML-service's prediction error while not changing the attacker's relative estimation error.

Our work, which was mainly analytical in nature, is a simple proof of concept and to remain tractable, our results were limited to simple functional form assumptions. Real ML-services are likely to be highly nonlinear (random forests and neural networks are two primary examples). An obvious extension to this proof-of-concept is to investigate how injecting noise impacts prediction and estimation in these large and complex models. While analytical results may be intractable, computational experiments may reveal how features of the garbling function, for example, impact the trade-off between prediction error and estimation error. This would include an example using a real data set.

We also assumed relatively simple attacker models. Therefore, an obvious extension to this work is a full game theoretic treatment of a strategic attacker and defender. In such a game, the defender would choose the garbling function and the attacker would form rational beliefs about the garbling function and choose its estimation strategy optimally, given its beliefs. This extension would both ground our method in a rigorous decision theoretic framework and also illuminate "worse case" scenarios by assuming an infinitely rational attacker. 


\section{References}

[1] W.H. Greene. Econometric Analysis. Pearson Education, 2003.

[2] Zvi Griliches and Jerry A Hausman. Errors in variables in panel data. Journal of econometrics, 31(1):93-118, 1986.

[3] Jason Hartford, Greg Lewis, Kevin Leyton-Brown, and Matt Taddy. Counterfactual prediction with deep instrumental variables networks. arXiv preprint arXiv:1612.09596, 2016.

[4] Matthew Jagielski, Nicholas Carlini, David Berthelot, Alex Kurakin, and Nicolas Papernot. High-fidelity extraction of neural network models. arXiv preprint arXiv:1909.01838, 2019.

[5] Mika Juuti, Sebastian Szyller, Samuel Marchal, and N Asokan. Prada: protecting against dnn model stealing attacks. arXiv preprint arXiv:1805.02628, 2018.

[6] Taesung Lee, Benjamin Edwards, Ian Molloy, and Dong Su. Defending against machine learning model stealing attacks using deceptive perturbations. arXiv preprint arXiv:1806.00054, 2018.

[7] Daniel Lowd and Christopher Meek. Adversarial learning. In Proceedings of the eleventh ACM SIGKDD international conference on Knowledge discovery in data mining, pages 641-647. ACM, 2005.

[8] Tribhuvanesh Orekondy, Bernt Schiele, and Mario Fritz. Knockoff nets: Stealing functionality of black-box models. In Proceedings of the IEEE Conference on Computer Vision and Pattern Recognition, pages 4954-4963, 2019.

[9] Nicolas Papernot, Patrick McDaniel, Ian Goodfellow, Somesh Jha, Z Berkay Celik, and Ananthram Swami. Practical black-box attacks against machine learning. In Proceedings of the 2017 ACM on Asia conference on computer and communications security, pages 506-519. ACM, 2017.

[10] Mauro Ribeiro, Katarina Grolinger, and Miriam AM Capretz. Mlaas: Machine learning as a service. In 2015 IEEE 14th International Conference on Machine Learning and Applications (ICMLA), pages 896-902. IEEE, 2015.

[11] Yi Shi, Yalin Sagduyu, and Alexander Grushin. How to steal a machine learning classifier with deep learning. In 2017 IEEE International Symposium on Technologies for Homeland Security (HST), pages 1-5. IEEE, 2017.

[12] Reza Shokri, Marco Stronati, Congzheng Song, and Vitaly Shmatikov. Membership inference attacks against machine learning models. In 2017 IEEE Symposium on Security and Privacy (SP), pages 3-18. IEEE, 2017.

[13] Florian Tramèr, Fan Zhang, Ari Juels, Michael K Reiter, and Thomas Ristenpart. Stealing machine learning models via prediction apis. In 25th $\{U S E N I X\}$ Security Symposium ( $\{U S E N I X\}$ Security 16), pages 601-618, 2016.

[14] Binghui Wang and Neil Zhenqiang Gong. Stealing hyperparameters in machine learning. In 2018 IEEE Symposium on Security and Privacy (SP), pages 36-52. IEEE, 2018. 
[15] Tom Wansbeek. Gmm estimation in panel data models with measurement error. Journal of Econometrics, 104(2):259-268, 2001.

[16] Xi Wu, Matthew Fredrikson, Somesh Jha, and Jeffrey F Naughton. A methodology for formalizing model-inversion attacks. In 2016 IEEE 29th Computer Security Foundations Symposium (CSF), pages 355-370. IEEE, 2016.

[17] Huadi Zheng, Qingqing Ye, Haibo Hu, Chengfang Fang, and Jie Shi. Bdpl: A boundary differentially private layer against machine learning model extraction attacks. In European Symposium on Research in Computer Security, pages 66-83. Springer, 2019. 